\title{
Ingestão de resíduos antropogênicos por tartarugas marinhas no litoral norte do estado da Bahia, Brasil
}

\author{
Anthropogenic debris ingestion by sea turtles in the northern coast of Bahia, Brazil

\begin{abstract}
Gustavo Rodamilans Macedo ${ }^{\mathrm{I}}$ Thaís Torres Pires ${ }^{\mathrm{II}}$ Gonzalo Rostán ${ }^{\mathrm{II}}$ Daphne Wrobel Goldberg ${ }^{\mathrm{III}}$ Danielle Custódio Leal ${ }^{\mathrm{I}}$ Américo Fróes Garcez Neto ${ }^{\mathrm{I}}$ Carlos Roberto Franke ${ }^{\mathrm{II}}$
\end{abstract}

\section{RESUMO}

Este trabalho descreve a presença de resíduos antropogênicos no trato digestório de tartarugas marinhas no Litoral Norte da Bahia, Brasil. Foram realizadas necropsias no trato digestório de 45 tartarugas marinhas encontradas mortas (Chelonia mydas $n=36$; Eretmochelys imbricata $n=9$ ), no período de janeiro de 2006 a outubro de 2007. Em 60\% (27/45) das tartarugas necropsiadas foram encontrados resíduos, especialmente aqueles relacionados à atividade de pesca. Os resíduos encontravam-se ao longo de todo o trato gastrointestinal, com predominância no intestino grosso. A ingestão de resíduos pelas tartarugas marinhas do Litoral Norte da Bahia pode levar a debilidade e até mesmo provocar a morte destes animais.

Palavras-chave: Chelonia mydas, Eretmochelys imbricata, lixo, necropsia.

\section{ABSTRACT \\ This study investigates the presence of anthropogenic debris in the digestive tract of sea turtles in the Northern Coast of Bahia, Brazil. Necropsies were performed on 45 turtles, 36 green turtles (Chelonia mydas) and 9 hawksbills (Eretmochelys imbricata), found dead between january 2006 and october 2007. Debris was found in 60\% of the animals, especially those related to fishing activities. Litter could be found throughout the entire gastrointestinal tract, but it was found predominantly in the large intestine $(47.53 \%)$. The ingestion of debris by turtles from the Northern coast of Bahia may lead these animals to starvation, weakness and even death.}

Key words: Chelonia mydas, Eretmochelys imbricata, debris, necropsy.

\section{INTRODUÇÃO}

A tartaruga verde, Chelonia mydas, e a tartaruga de pente, Eretmochelys imbricata, são consideradas em perigo de extinção e em perigo crítico de extinção, respectivamente (IUCN, 2007). As atuais principais ameaças às populações de tartarugas marinhas têm origem antrópica, a exemplo da destruição das áreas de nidificação; da poluição dos mares; da captura incidental de tartarugas; além de outras práticas não sustentáveis (HAMANN et al., 2010). MARCOVALDI \& MARCOVALDI (1999) relataram que o litoral norte do estado da Bahia é uma importante área de alimentação das tartarugas verdes juvenis e de nidificação das tartarugas de pente e, por conseguinte, essas espécies estão sujeitas aos impactos ambientais produzidos nesta região.

BJORNDAL et al. (1994) descrevem os efeitos causados pela ingestão de resíduos antrópicos por tartarugas marinhas e relatam a possibilidade destes causarem a obstrução do trato digestório, causando a morte do animal, mesmo quando ingeridos

'Programa de Pós-graduação em Ciência Animal nos Trópicos, Universidade Federal da Bahia (UFBA), 40170-110, Salvador, BA, Brasil. E-mail: gustavorodamilans@ hotmail.com. *Autor para correspondência.

"Fundação Centro Brasileiro de Proteção e Pesquisa das Tartarugas Marinhas (Fundação Pro-Tamar), Salvador, BA, Brasil.

IIIFundação Centro Brasileiro de Proteção e Pesquisa das Tartarugas Marinhas (Fundação Pró-Tamar), Florianópolis, SC, Brasil.

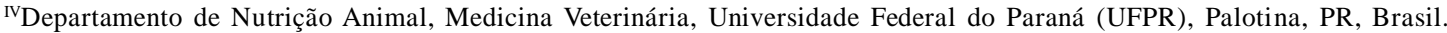

`Departamento de Produção Animal, Escola de Medicina Veterinária e Zootecnia, Universidade Federal da Bahia (UFBA), Salvador, BA, Brasil. 
em pequena quantidade. BUGONI et al. (2001), em estudo realizado no Sul do Brasil, observaram a presença de resíduos antropogênicos no estômago de $60,5 \%$ (23/38) das tartarugas marinhas examinadas e sugeriram que os resíduos encontrados eram originários, não só da atividade pesqueira, mas também oriundos da atividade turística nas praias da região.

Segundo BJORNDAL (2000), é importante determinar a ingestão de resíduos antropogênicos por tartarugas marinhas nas áreas de alimentação, as quais são consideradas prioritárias para o manejo populacional e para a sobrevivência dessas espécies de quelônios.

A ingestão de resíduos sólidos antropogênicos por tartarugas marinhas é descrita em outros países, porém, no litoral brasileiro, os estudos ainda são insuficientes (BUGONI et al., 2001) e, na área deste trabalho, ainda não há relatos sobre o tema. Os principais objetivos deste estudo foram avaliar, dentre os casos de morte de tartarugas registrados e necropsiados pelo TAMAR/ICMBio no litoral norte da Bahia, a frequência de achado de resíduos antropogênicos no trato digestório desses animais, descrever sua localização no trato e identificar o material e sua possível origem.

\section{MATERIAL E MÉTODOS}

No período de janeiro de 2006 a outubro de 2007, foram realizadas necropsias em 45 tartarugas marinhas das espécies $\boldsymbol{C}$. mydas $(\mathrm{n}=36) \boldsymbol{e} \boldsymbol{E}$. imbricata $(\mathrm{n}=9)$, encontradas mortas ou que vieram a óbito, durante tratamento no Setor Veterinário do Projeto TAMAR/Praia do Forte. Esses animais foram encontrados pelas equipes do Projeto TAMAR locadas estrategicamente em quatro bases, cobrindo cerca de $212 \mathrm{~km}$ do litoral norte da Bahia (ponto mais ao sul $12^{\circ} 58^{\prime} 40^{\prime \prime} \mathrm{S} x-38^{\circ} 25^{\prime} 14^{\prime \prime} \mathrm{W}$ e o ponto mais ao norte $11^{\circ} 27^{\prime} 53^{\prime \prime} \mathrm{S}$ x $-37^{\circ} 21^{\prime} 06^{\prime \prime} \mathrm{W}$ ), abrangendo as praias de Arembepe, Praia do Forte, Sauípe, e Sítio do Conde. Das 45 tartarugas marinhas, 21 (46,7\%) foram encontradas na região de Arembepe, 12 (26,7\%) na Praia do Forte, sete $(15,5 \%)$ em Sauípe e cinco $(11,1 \%)$ em Sítio do Conde (Figura 1).

Todas as 45 tartarugas marinhas foram identificadas quanto à espécie, medidas, pesadas e verificadas o estado corporal. O método de medição foi realizado de acordo com BOLTEN (2000) e o estado corporal foi classificado de acordo com a observação clínica do animal, utilizada como rotina do Projeto TAMAR, em: caquético, ruim, regular e bom.

Para pesquisar a presença de material antropogênico, foi realizada a necropsia do trato digestório das tartarugas, iniciando no esôfago e indo até a porção distal do intestino grosso. Os resíduos encontrados foram classificados quanto ao tipo de material, possível origem pesqueira e localização no trato digestório (esôfago, estômago, intestino delgado e intestino grosso).

Para a análise dos dados de ocorrência dos resíduos antropogênicos no trato digestório das tartarugas marinhas, foram calculadas suas frequências e utilizado o teste do Qui-quadrado para comparar as frequências de resíduos nos diferentes compartimentos do trato digestório.

\section{RESULTADOS E DISCUSSÃO}

As necropsias revelaram a presença de material antropogênico em 60\% (27/45) das tartarugas, sendo 20 exemplares da espécie $\boldsymbol{C}$. mydas e sete da espécie $\boldsymbol{E}$. imbricata. Foi observado que a maioria dos animais com resíduos no trato digestório apresentava comprometimento do estado corporal, apesar da quantidade de resíduo encontrada em alguns espécimes não ser suficiente para ser considerada, macroscopicamente, como causa mortis. Foi observado também que 46,7\% (21/45) dos animais foram classificados, de acordo com as observações clínicas, como caquéticos (Tabela 1).

As $\boldsymbol{C}$. mydas apresentaram comprimento curvilíneo médio de carapaça (CCC) de $36,14 \mathrm{~cm}$, com CCC máximo de 104,0cm e mínimo de $8,5 \mathrm{~cm}$, com peso médio de $3,6 \mathrm{~kg}$, enquanto as $\boldsymbol{E}$. imbricata tiveram CCC médio de $32,87 \mathrm{~cm}$, com CCC máximode $57,0 \mathrm{~cm}$ e mínimo de $11,5 \mathrm{~cm}$ e peso médio de $3,77 \mathrm{~kg}$. Com exceção de um exemplar de $\boldsymbol{C}$. mydas adulta de $104 \mathrm{~cm}$ de CCC, os demais eram filhotes ou juvenis.

Predominaram os resíduos de origem pesqueira 62,9\% (17/27) como: fios de nylon, usados na pesca e na confecção de redes de emalhe, bem como cordas de nylon usadas para sustentar redes de pesca e para amarrações em barcos. Os 37,1\% (10/27) restantes foram resíduos diversos, tais como sacos plásticos; pedaços de plástico duro; isopor; corda de sisal; filtros de cigarros; pedaços de canudos plásticos dentre outros.

Os resíduos antropogênicos foram encontrados em todos os quatro compartimentos do trato digestório, observando-se a seguinte frequência decrescente de achados: intestino grosso 46,7\% (21/ 45); estômago 31,1\% (14/45); intestino delgado 20,0\% (9/45) e esôfago 11,73\% (8/45). Utilizando o teste Quiquadrado, observou-se que há diferença significativa entre os resultados de frequência de resíduos antropogênicos de cada compartimento do trato digestório das tartarugas marinhas (valor de $\mathrm{P}>0,05$ ). 


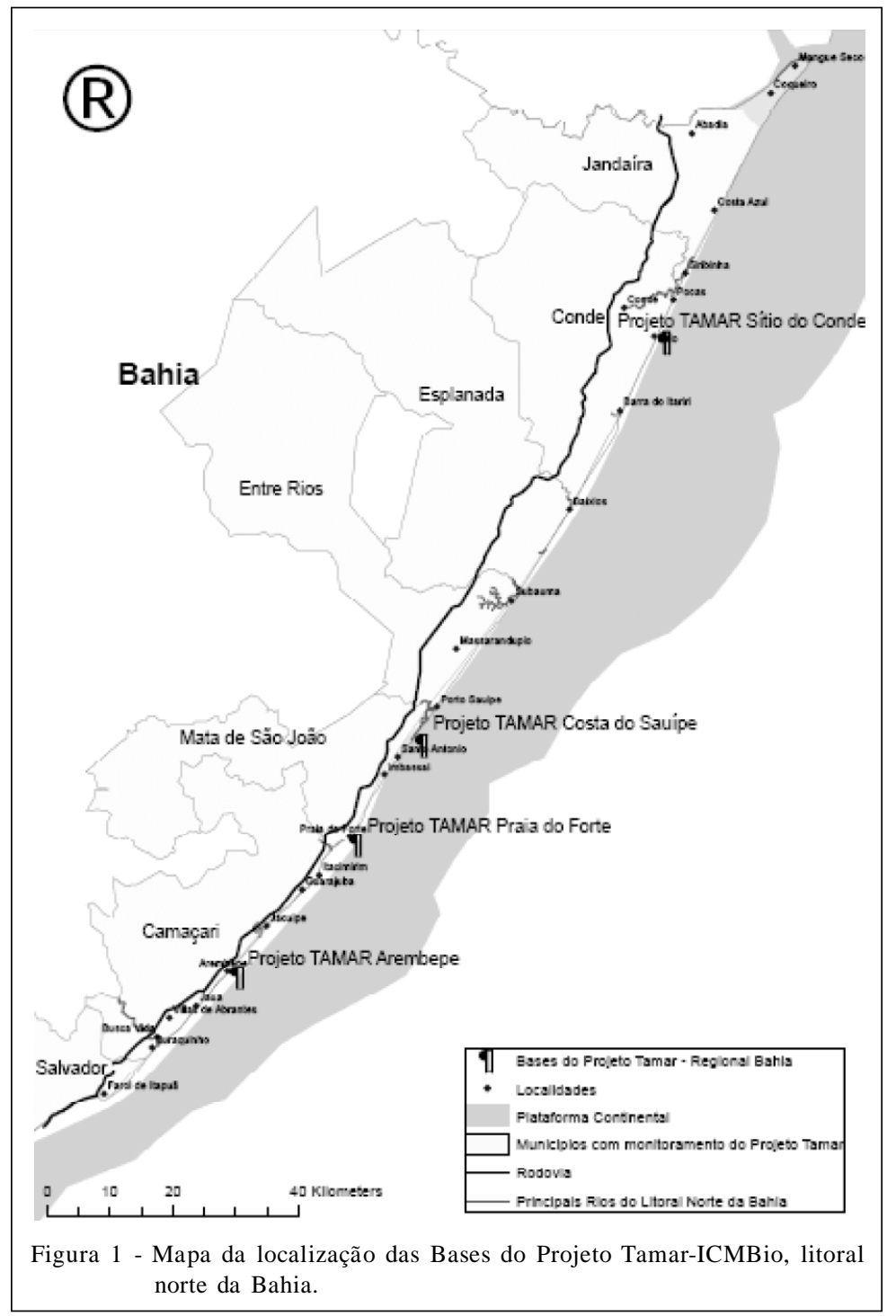

A frequência de resíduos antropogênicos no trato digestório de tartarugas marinhas é relatada em diversas regiões oceânicas: $20 \%$ no Mediterrâneo Central (GRAMENTZ, 1988); 56\% na Costa da Flórida (BJORNDAL et al., 1994); 79,6\% no Mediterrâneo Ocidental (TOMÁS et al., 2002); 60,5\% no Atlântico Sul (BUGONI et al., 2001) e 19,35\% nas Ilhas Canárias (ORÓS et al., 2005). No presente estudo, registrou-se a elevada presença $(60 \%)$ de resíduos antropogênicos ingeridos por tartarugas marinhas no litoral norte do estado da Bahia. GRAMENTZ (1988) sugere que esses animais ingerem equivocadamente os resíduos, porque os confundem com alimentos naturais, como águasvivas e peixes. SCHULMAN \& LUTZ (1995) confirmaram que tartarugas marinhas, quando com fome, alimentam-se de resíduos e TOMÁS et al. (2002) sugerem que as tartarugas marinhas apresentam uma baixa seletividade ao se alimentarem. Segundo MARCOVALDI \& MARCOVALDI(1999), o litoral norte da Bahia é uma importante área de nidificação, sendo a Praia do Forte (Figura 1) uma área mista de nidificação e alimentação. Porém, a presença constante de animais da espécie $\boldsymbol{C}$. mydas e $\boldsymbol{E}$. imbricata na área de Arembepe sugere também sua importância como área de alimentação, uma vez que apresenta variedade de alimentos, a exemplo de algas do gênero Sargassum spp. que servem de alimento para tartarugas na fase juvenil. Segundo SCHULMAN \& LUTZ (1995), o tempo normal de excreção do alimento é de seis a sete dias, sendo que, quando ocorre a ingestão de resíduos, esse tempo de excreção aumenta para, no mínimo, 14 dias. De acordo com essa informação, e considerando o 
Tabela 1 - Relação dos animais necropsiados, condição corporal, presença, local e tipo do resíduo.

\begin{tabular}{|c|c|c|c|c|c|c|c|c|}
\hline \multirow[b]{2}{*}{ C. mydas $(\mathrm{n}=36)$} & \multirow{3}{*}{$\begin{array}{c}\begin{array}{c}\text { Condição } \\
\text { corporal }\end{array} \\
1\end{array}$} & \multicolumn{2}{|c|}{ Presença de resíduo } & \multicolumn{4}{|c|}{-------------'Local dos resíduos-------------- } & \multirow{2}{*}{$\begin{array}{c}\text { Tipo de resíduo } \\
\text { Material }\end{array}$} \\
\hline & & Sim & Não & Esôfago & Estômago & ID & IG & \\
\hline & & $\mathrm{X}$ & & & & & $\mathrm{X}$ & náilon, plástico rígido \\
\hline & 1 & $\mathrm{X}$ & & $\mathrm{X}$ & $\mathrm{X}$ & & & plástico, pedras \\
\hline & 1 & $\mathrm{X}$ & & $\mathrm{X}$ & $\mathrm{X}$ & & & plástico \\
\hline & 1 & $X$ & & $\mathrm{X}$ & $\mathrm{X}$ & $\mathrm{X}$ & $\mathrm{X}$ & plástico \\
\hline & 1 & $\mathrm{X}$ & & & $\mathrm{X}$ & $\mathrm{X}$ & $\mathrm{X}$ & palha \\
\hline & 1 & $\mathrm{X}$ & & $\mathrm{X}$ & $\mathrm{X}$ & & $\mathrm{X}$ & plástico, náilon \\
\hline & 1 & $\mathrm{X}$ & & $\mathrm{X}$ & & & $\mathrm{X}$ & plástico \\
\hline & 1 & $\mathrm{X}$ & & & $\mathrm{X}$ & & $\mathrm{X}$ & plástico \\
\hline & 1 & $\mathrm{X}$ & & $\mathrm{X}$ & $\mathrm{X}$ & & & náilon, corda \\
\hline & 1 & $\mathrm{X}$ & & & & & $\mathrm{X}$ & náilon, corda, plástico \\
\hline & 1 & $\mathrm{X}$ & & & & $\mathrm{X}$ & $X$ & plástico, náilon \\
\hline & 1 & $\mathrm{X}$ & & & & & $\mathrm{X}$ & plástico flexível, náilon \\
\hline & 1 & & $\mathrm{X}$ & & & & & - \\
\hline & 1 & & $\mathrm{X}$ & & & & & - \\
\hline & 1 & & $\mathrm{X}$ & & & & & - \\
\hline & 1 & & $\mathrm{X}$ & & & & & - \\
\hline & 1 & & $\mathrm{X}$ & & & & & - \\
\hline & 2 & $\mathrm{X}$ & & & & & $X$ & náilon \\
\hline & 2 & $\mathrm{X}$ & & & & & $\mathrm{X}$ & plástico, náilon \\
\hline & 2 & $\mathrm{X}$ & & & $\mathrm{X}$ & & $\mathrm{X}$ & plástico, náilon \\
\hline & 2 & $\mathrm{X}$ & & & & $\mathrm{X}$ & $\mathrm{X}$ & $\begin{array}{l}\text { plástico rígido e flexível, } \\
\text { náilon, corda }\end{array}$ \\
\hline & 2 & $\mathrm{X}$ & & & & $\mathrm{X}$ & & náilon \\
\hline & 2 & $\mathrm{X}$ & & & & & $X$ & plástico, náilon \\
\hline & 2 & & $\mathrm{X}$ & & & & & - \\
\hline & 2 & & $\mathrm{X}$ & & & & & - \\
\hline & 2 & & $\mathrm{X}$ & & & & & - \\
\hline & 2 & & $\mathrm{X}$ & & & & & - \\
\hline & 3 & $\mathrm{X}$ & & & & & $\mathrm{X}$ & não identificado \\
\hline & 3 & & $\mathrm{X}$ & & & & & - \\
\hline & 4 & $\mathrm{X}$ & & & & $\mathrm{X}$ & $X$ & náilon \\
\hline & 4 & & $\mathrm{X}$ & & & & & - \\
\hline & 4 & & $\mathrm{X}$ & & & & & - \\
\hline & 4 & & $\mathrm{X}$ & & & & & - \\
\hline & 4 & & $\mathrm{X}$ & & & & & - \\
\hline & 4 & & $\mathrm{X}$ & & & & & - \\
\hline & 4 & & $\mathrm{X}$ & & & & & - \\
\hline \multicolumn{9}{|l|}{$\begin{array}{l}\text { E. imbricata } \\
(\mathrm{N}=9)\end{array}$} \\
\hline & 1 & $X$ & & & $\mathrm{X}$ & & & plástico, náilon \\
\hline & 1 & $\mathrm{X}$ & & & & $\mathrm{X}$ & $\mathrm{X}$ & plástico, areia \\
\hline & 1 & $\mathrm{X}$ & & $\mathrm{X}$ & $\mathrm{X}$ & & $\mathrm{X}$ & plástico, náilon, pedras \\
\hline & 1 & $\mathrm{X}$ & & $X$ & $X$ & $\mathrm{X}$ & $X$ & plástico \\
\hline & 1 & $\mathrm{X}$ & & & $\mathrm{X}$ & $\mathrm{X}$ & $\mathrm{X}$ & plástico, náilon \\
\hline & 2 & $\mathrm{X}$ & & & $\mathrm{X}$ & & $X$ & plástico, náilon \\
\hline & 3 & $\mathrm{X}$ & & & $\mathrm{X}$ & & & plástico \\
\hline & 3 & & $\mathrm{X}$ & & & & & - \\
\hline & 3 & & $\mathrm{X}$ & & & & & - \\
\hline
\end{tabular}

1: caquético; 2: ruim; 3: regular; 4: bom; ID: intestino delgado; IG: intestino grosso. 
estado de morte recente dos animais analisados neste estudo, é provável que os resíduos encontrados no trato digestório tenham sido ingeridos na própria região abrangida nesta pesquisa e, considerando as características dos resíduos encontrados (fios de nylon, sacos plásticos, dentre outros), é possível sugerir que estes tenham sua origem também local, oriundos da atividade pesqueira de comunidades litorâneas ou ainda das atividades turísticas (Figura 2).

BJORNDAL et al. (1994) afirmam que a observação do trato digestório deve ser realizada desde o esôfago até o intestino grosso para que a frequência de resíduos encontrados não seja subestimada. Essa recomendação se apoia nos dados dos próprios autores, que registraram a ingestão de material antropogênico em 56\% (24/43) das tartarugas que tiveram todo o trato digestório inspecionado e apenas $14 \%$ (6/43) quando o exame se restringiu ao esôfago e estômago. SCHULMAN \& LUTZ (1995) sugerem que, em virtude das numerosas curvaturas do intestino das tartarugas, há maior chance de encontrar resíduos nessa porção do trato digestório.

Neste estudo, foi observada a presença de resíduos em todos os quatro compartimentos gástricos, sendo que no intestino grosso se concentrou a maior frequência dos achados: 46,7\% (21/45). O intestino grosso foi o único compartimento que apresentou diferença significativa na frequência relativa dos achados, quando comparado com os demais compartimentos analisados, o que corrobora a observação feita por BJORNDAL et al. (1994).

Foram encontrados materiais antropogênicos nos animais classificados nas quatro categorias de estado corporal, sendo a maior frequência observada nos animais com estado corporal caquético. Considerando que os animais eram de vida livre, não há como afirmar se o início do processo de decaimento da condição corporal das tartarugas se deva à ingestão de resíduos, conduzindo-as a um posterior estado caquético, em virtude de complicações digestórias, ou se a ingestão de materiais antropogênicos ocorra em consequência de um estado debilitado prévio, decorrente de uma infecção, intoxicação ou algum tipo de lesão corporal que dificulte a busca por alimentos de sua dieta natural, que implique um esforço físico de natação e mergulho. $\mathrm{O}$ estado debilitado e a fome estariam levando o animal a ingerir materiais antropogênicos devido ao menor esforço despendido na sua obtenção.

Apesar de outros estudos apontarem o plástico como principal resíduo antropogênico ingerido pelas tartarugas marinhas (BJORNDAL et al., 1994; BUGONI et al., 2001; TOMÁS et al., 2002; ORÓS et al., 2005), no presente trabalho, observou-se que resíduos

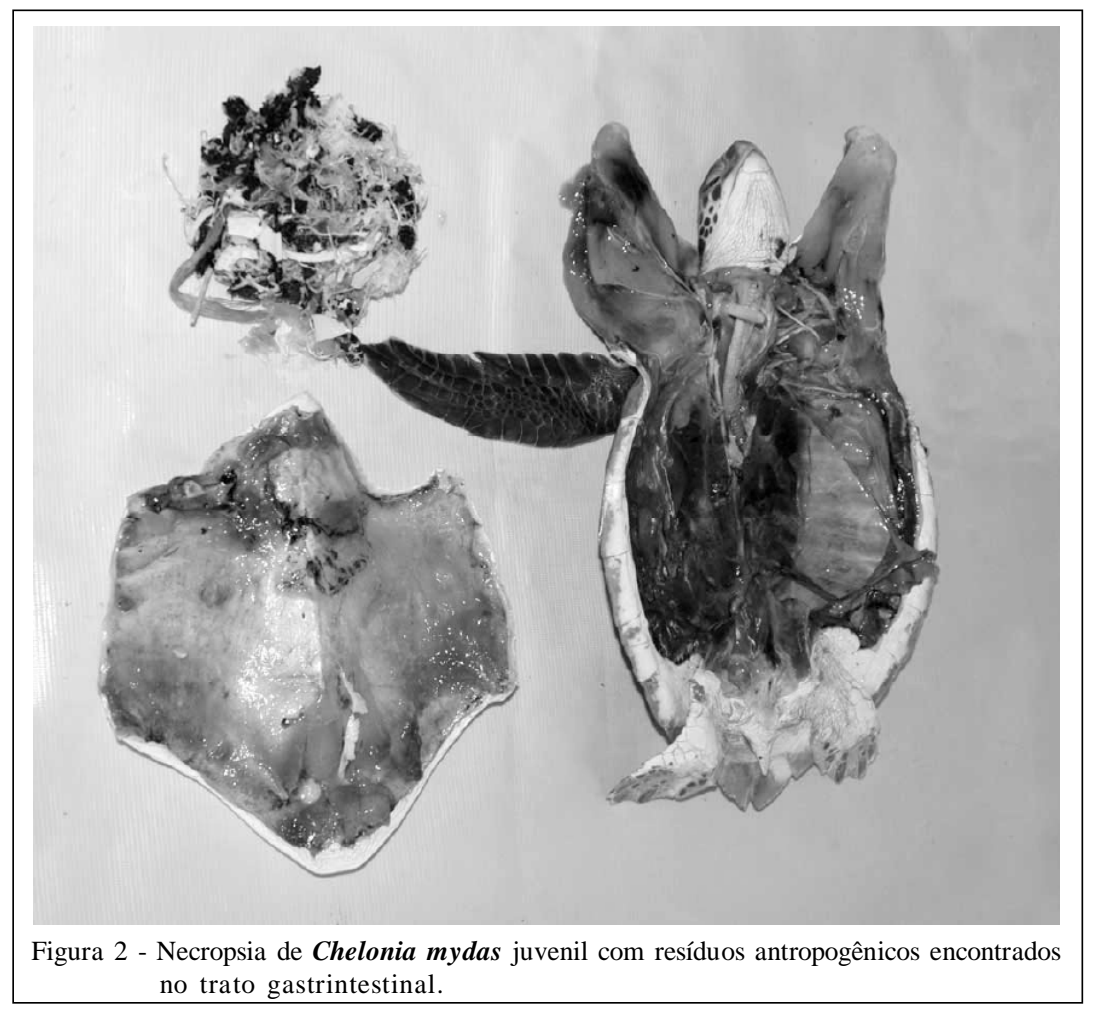

Ciência Rural, v.41, n.11, nov, 2011. 
antropogênicos de origem pesqueira, como linhas de nylon e cordas de embarcação, foram encontrados em $62,9 \%$ dos casos. De acordo com BJORNDAL et al. (1994), a ingestão de linhas de nylon pode provocar a morte do animal se interferir na função normal do trato digestório, impedindo a movimentação da digesta, causando impactação e provocando volvos gástricos e intestinais.

\section{CONCLUSÃO}

As tartarugas marinhas que habitam o litoral norte da Bahia ingerem resíduos antropogênicos de origem pesqueira ou não, tendo como consequência o comprometimento da saúde e, em alguns casos, a morte.

Estes resultados reforçam a necessidade de um esforço abrangente de conscientização ambiental, não só das populações litorâneas, como também dos atores envolvidos na atividade turística. São necessários projetos multidisciplinares de educação ambiental que venham a minimizar a disposição de resíduos antropogênicos no meio, reduzindo o risco de ingestão destes pelas tartarugas marinhas que buscam nesta região um refugio para alimentação e nidificação.

\section{REFERÊNCIAS}

BJORNDAL, K.A. Prioridad em la pesquisa em áreas de alimentacíon. In. ECKERT, K.L. et al. Técnicas de investigación y manejo para la conservación de las tortugas marinas. Trad. Raquel Briseño-Dueñas. et.al. Pennsylvania: IUCN/CSE, 2000. p.13-15. (IUCN/SSC Marine Turtle Specialist Group). Disponível em: <http:// mtsg.files.wordpress.com/2010/07/04-prioridades-para-lainvestigacion-en-habitats-de-alimentacion.pdf >. Acesso em: 18 dez. 2010.

BJORNDAL, K.A. et al. Ingestion of marine debris by juvenile sea turtles in coastal Florida habitats. Marine Pollution Bulletin, v.28, n.3, p.154-158, 1994. Disponível em: <http:/ / a c c s t r. u f l. e d u / p u b l i c a t i o n s / BjorndalEt A1_1994_Ingestion OfMarine Deb risByJuvenileSeaTurtlesInCostalFlorida.pdf $>$. Acesso em: 20 maio, 2007. doi: 10.1016/0025-326X(94)90391-3.

BOLTEN, A.B. Técnicas para la medición de tortugas marinas. Técnicas de investigación y manejo para la conservación de las tortugas marinas. Trad. Raquel Briseño-Dueñas. et. al. Pennsylvania: IUCN/CSE, 2000. p.126-131. (IUCN/SSC Marine Turtle Specialist Group). Disponível em: <http:// mtsg.files.wordpress.com/2010/07/18-tecnicas-para-la-medirde-tortugas-marinas.pdf>. Acesso em: 18 dez. 2010.

BUGONI, L. et al. Marine debris and human impacts on sea turtles in Southern Brazil. Marine Pollution Bulletin, v.42, n.12, p.1330-1334, 2001. Disponível em: <http:// www.seaturtle.org/PDF/BugoniL_2001_MarPollutBull.pdf $>$. Acesso em: 20 maio, 2007. doi: 10.1016/S0025326X(01)00147-3.

GRAMENTZ, D. Involvement of loggerhead turtle with the plastic, metal, and hydrocarbon pollution in the Central Mediterranean. Marine Pollution Bulletin, v.19, n.1, p.1113, 1988. Disponível em: <http://www.sciencedirect.com/ science/article/pii/0025326X88907461>. Acesso em: 20 maio, 2007. doi: 10.1016/0025-326X(88)90746-1.

HAMANN, M. et al. Global research priorities for sea turtles: informing management and conservation in the 21 st century. Endangered Species Research, v.11, p.245-269, 2010. Disponível em: <http://accstr.ufl.edu/publications/ Hamann_et_al_ESR_2010.pdf $>$. Acesso em: 15 out. 2011. doi: $10.3354 /$ esr0027.

IUCN. IUCN red list of threatened species 2007. Disponível em: 〈http://www.iucnredlist.org >. Acesso em: 10 out. 2007.

MARCOVALDI, M.A.; MARCOVALDI, G.G. Marine turtles of Brazil: the history and structure of projeto TAMAR-IBAMA. Biological Conservation, v.93, p.35-41, 1999. Disponível em: < h t t p : / / w w w. s e a t u r t l e o o g / P D F / Marcovaldi_1999_BiolConserv.pdf >. Acesso em: 20 maio, 2007. doi:10.1016/S0006-3207(99)00043-9.

ORÓS, J. et al. Diseases and causes of mortality among sea turtles stranded in the Canary Islands, Spain (1998-2001). Diseases of aquatic organisms, v.63, p.13-24, 2005. Disponível em: <http://www.int-res.com/articles/dao2005/63/ d063p013.pdf>. Acesso em: 20 maio, 2007.

SCHULMAN, A.A.; LUTZ, P. The effect of plastic ingestion on lipid metabolism in the green sea turtle (Chelonia mydas). In: ANNUAL WORKSHOP ON SEA TURTLE BIOLOGY AND CONSERVATION, 12., 1992, Georgia. Proceedings... Flórida: NOAA, 1995. p.122-124. NOAA Technical Memorandum (NMFS-SEFSC-361).

TOMÁS, J. et al. Marine debris ingestion in loggerhead sea turtles, Caretta caretta, from the Western Mediterranean. Marine Pollution Bulletin, v.44, p.211-216, 2002. Disponível em: < h t t p : / / w w w. s e a t u r t 1 e o o g / P D F / TomasJ_2002a_MarPollBull.pdf>. Acesso em: 22 maio, 2007. doi: $10.1016 /$ S0025-326X(01)00236-3. 